\title{
ROTHE TIME-DISCRETIZATION METHOD FOR THE SEMILINEAR HEAT EQUATION SUBJECT TO A NONLOCAL BOUNDARY CONDITION
}

\author{
NABIL MERAZGA AND ABDELFATAH BOUZIANI
}

Received 24 February 2006; Revised 28 May 2006; Accepted 29 May 2006

This paper is devoted to prove, in a nonclassical function space, the weak solvability of a mixed problem which combines a Neumann condition and an integral boundary condition for the semilinear one-dimensional heat equation. The investigation is made by means of approximation by the Rothe method which is based on a semidiscretization of the given problem with respect to the time variable.

Copyright (c) 2006 N. Merazga and A. Bouziani. This is an open access article distributed under the Creative Commons Attribution License, which permits unrestricted use, distribution, and reproduction in any medium, provided the original work is properly cited.

\section{Introduction}

In our earlier work [5], an investigation was made for an initial-boundary value problem with an integral condition for the two-dimensional diffusion equation. There, a suitable transformation has allowed us to bring the considered problem back to an equivalent problem of the following form:

$$
\begin{gathered}
\frac{\partial u}{\partial t}-\frac{\partial^{2} u}{\partial x^{2}}=f(x, t), \quad(x, t) \in(0,1) \times[0, T], \\
u(x, 0)=U_{0}(x), \quad x \in(0,1), \\
\frac{\partial u}{\partial x}(0, t)=\alpha(t), \quad t \in[0, T], \\
\int_{0}^{1} u(x, t) d x=E(t), \quad t \in[0, T]
\end{gathered}
$$

whose weak solvability was then proved with the help of the Rothe time-discretization method.

In the present paper, we consider a generalization of problem (1.1), namely the problem of finding a function $v=v(x, t)$ which obeys, in a weak sense, the semilinear diffusion 
2 A semilinear heat equation with a nonlocal condition

equation

$$
\frac{\partial v}{\partial t}-\frac{\partial^{2} v}{\partial x^{2}}=\mathrm{f}(x, t, v), \quad(x, t) \in(0,1) \times[0, T]
$$

subject to the initial condition

$$
v(x, 0)=V_{0}(x), \quad x \in[0,1]
$$

the Neumann condition

$$
\frac{\partial v}{\partial x}(0, t)=g(t), \quad t \in[0, T]
$$

and the integral boundary condition

$$
\int_{0}^{1} v(x, t) d x=E(t), \quad t \in[0, T],
$$

where f, $V_{0}, g$, and $E$ are given functions, and $T$ is a positive constant.

The method used here to investigate problem (1.2)-(1.5) is the same as in [5], the socalled "Rothe method." However, the presence of the semilinearity in (1.2) complicates the process of derivating the necessary a priori estimates and proving the convergence of the method. Moreover, we follow in Section 5 a slightly different way which is simpler and shorter than the one in [5].

It is interesting to note that problem (1.2)-(1.5) has, like (1.1), many practical interpretations in the context of chemical engineering, thermoelasticity, heat conduction theory, population dynamics, and so forth (see the references in [5]).

Introducing a new unknown function $u$ by setting

$$
u(x, t)=v(x, t)-r(x, t)
$$

where

$$
r(x, t)=g(t)\left(x-\frac{1}{2}\right)+E(t)
$$

it clearly follows that $u$ satisfies the following problem:

$$
\begin{gathered}
\frac{\partial u}{\partial t}-\frac{\partial^{2} u}{\partial x^{2}}=f(x, t, u), \quad(x, t) \in(0,1) \times I, \\
u(x, 0)=U_{0}(x), \quad x \in[0,1], \\
\frac{\partial u}{\partial x}(0, t)=0, \quad t \in I, \\
\int_{0}^{1} u(x, t) d x=0, \quad t \in I,
\end{gathered}
$$


where $I$ stands for the time interval $[0, T]$ and

$$
\begin{gathered}
f(x, t, u):=\mathrm{f}(x, t, u+r)-\frac{\partial r(x, t)}{\partial t}, \\
U_{0}(x):=V_{0}(x)-r(x, 0) .
\end{gathered}
$$

Hence, instead of studying directly the problem (1.2)-(1.5), we concentrate our attention on problem (1.8)-(1.11). Once $u$ is known, the function $v$ is immediately obtained through the relation $v=u+r$.

The plan of the paper is as follows. In Section 2, notations, assumptions on data, and some useful results are given before stating the precise sense of the required solution as well as the main result of the paper. In Section 3, a semidiscretization in time of problem (1.8)-(1.11) is performed to construct approximate solutions, the so-called "Rothe approximations." Some necessary a priori estimates for these approximations are derived in Section 4, and then used, in Section 5, to establish a convergence and existence result for the problem under study.

\section{Preliminaries}

In the course of the paper, $(\cdot, \cdot)$ denotes the usual scalar product in $L^{2}(0,1)$ and $\|\cdot\|$ the corresponding norm, while $H^{2}(0,1)$ denotes the usual (real) second-order Sobolev space on $(0,1)$ with norm $\|\cdot\|_{H^{2}(0,1)}$. Let $V$ be the set which we define as follows:

$$
V:=\left\{\phi \in L^{2}(0,1) ; \int_{0}^{1} \phi(x) d x=0\right\} .
$$

Clearly, $V$ is a Hilbert space for $(\cdot, \cdot)$.

In addition to the standard functional spaces of the types $C(I, X), C^{0,1}(I, X), L^{2}(I, X)$, and $L^{\infty}(I, X)$ of continuous, Lipschitz-continuous, $L^{2}$-Bochner integrable, and essentially bounded functions from $I$ into a Banach space $X$, respectively (see, e.g., [4]), our analysis requires also the use of the nonclassical function space $B_{2}^{1}(0,1)$ introduced by the second author (see, e.g., $[1,2])$ as the completion of the space $C_{0}(0,1)$ of real continuous functions with compact support in $(0,1)$ with respect to the inner product

$$
(u, v)_{B_{2}^{1}}=\int_{0}^{1} \mathfrak{J}_{x} u \cdot \mathfrak{J}_{x} v d x
$$

where $\mathfrak{I}_{x} v=\int_{0}^{x} v(\xi) d \xi$ for every fixed $x \in(0,1)$. If $\|\cdot\|_{B_{2}^{1}}$ denotes the corresponding norm, that is,

$$
\|v\|_{B_{2}^{1}}=\sqrt{(v, v)_{B_{2}^{1}}}
$$

then, the inequality

$$
\|v\|_{B_{2}^{1}}^{2} \leqslant \frac{1}{2}\|v\|^{2}
$$

holds for every $v \in L^{2}(0,1)$, and hence the embedding $L^{2}(0,1) \rightarrow B_{2}^{1}(0,1)$ is continuous. 
4 A semilinear heat equation with a nonlocal condition

We should note that any given real function $\theta(x, t)$ on $(0,1) \times I$ is automatically identified with the corresponding abstract function $t \rightarrow \theta(t)=\theta(\cdot, t)$ defined from $I$ into some functional space on $(0,1)$ by setting $(\theta(t))(x)=\theta(x, t)$ for $x \in(0,1)$.

Strong and weak convergence are denoted by $\rightarrow$ or $\rightarrow$, respectively, and the symbol $c$ will stand for generic positive constants which may be different in the same discussion.

At several places, we will use the following continuous and discrete forms of Gronwall lemma.

LeMma 2.1. (i) Let $x(t) \geqslant 0, h(t), y(t)$ be real integrable functions on the interval $[a, b]$. If

$$
y(t) \leqslant h(t)+\int_{a}^{t} x(\tau) y(\tau) d \tau, \quad \forall t \in[a, b]
$$

then

$$
y(t) \leqslant h(t)+\int_{a}^{t} h(\tau) x(\tau) \exp \left(\int_{\tau}^{t} x(s) d s\right) d \tau, \quad \forall t \in[a, b]
$$

In particular, if $x(\tau) \equiv C$ is a constant and $h(\tau)$ is nondecreasing, then

$$
y(t) \leqslant h(t) e^{C(t-a)}, \quad \forall t \in[a, b] .
$$

(ii) Let $\left\{a_{i}\right\}$ be a sequence of real nonnegative numbers satisfying

$$
\begin{gathered}
a_{1} \leqslant A, \\
a_{i} \leqslant A+B h \sum_{k=1}^{i-1} a_{k}, \quad \forall i=2, \ldots,
\end{gathered}
$$

where $A, B$, and h are positives constants. Then

$$
a_{i} \leqslant A e^{B(i-1) h}, \quad \forall i=1,2, \ldots
$$

Proof. The proof of assertion (i) is the same as in [3, Lemma 1.3.19]. As for assertion (ii), it suffices to see that from our hypothesis, the following estimate follows:

$$
a_{i} \leqslant A(1+B h)^{i-1}, \quad \forall i=1,2, \ldots
$$

Indeed, we have first $a_{1} \leqslant A$ and $a_{2} \leqslant A+a_{1} B h \leqslant A(1+B h)$. Next, let us suppose that $a_{k} \leqslant A(1+B h)^{k-1}$ holds for all $k=1, \ldots, i-1$, then

$$
\begin{aligned}
a_{i} & \leqslant A+B h \sum_{k=1}^{i-1} A(1+B h)^{k-1}=A\left[1+B h \sum_{k=1}^{i-1}(1+B h)^{k-1}\right] \\
& =A\left[1+B h \frac{1-(1+B h)^{i-1}}{1-(1+B h)}\right]=A(1+B h)^{i-1} .
\end{aligned}
$$

Hence, using the elementary inequality $1+t \leqslant e^{t}$, for all $t \in \mathbb{R}_{+}$, we have $a_{i} \leqslant A e^{B(i-1) h}$, which was to be proved. 
Also, the elementary Cauchy inequality

$$
\alpha \beta \leqslant \frac{\varepsilon}{2} \alpha^{2}+\frac{1}{2 \varepsilon} \beta^{2}, \quad \forall \alpha, \beta \in \mathbb{R}, \forall \varepsilon \in \mathbb{R}_{+}^{*},
$$

will be useful to us thereafter.

Throughout the paper, we assume that

(H1) $f(t, w) \in L^{2}(0,1)$ for each pair $(t, w) \in I \times L^{2}(0,1)$ and the Lipschitz condition

$$
\left\|f(t, w)-f\left(t^{\prime}, w^{\prime}\right)\right\|_{B_{2}^{1}} \leqslant l\left[\left|t-t^{\prime}\right|\left(1+\|w\|_{B_{2}^{1}}+\left\|w^{\prime}\right\|_{B_{2}^{1}}\right)+\left\|w-w^{\prime}\right\|_{B_{2}^{1}}\right],
$$

for all $t, t^{\prime} \in I$, for all $w, w^{\prime} \in V$, holds for some positive constant $l$;

(H2) $U_{0} \in H^{2}(0,1)$;

(H3) $\left(d U_{0} / d x\right)(0)=0$ and $\int_{0}^{1} U_{0}(x) d x=0$, for consistency.

We will be concerned with a weak solution in the following sense.

Definition 2.2. Under a weak solution of problem (1.8)-(1.11), a function $u: I \rightarrow L^{2}(0,1)$ is understood such that

(i) $u \in L^{\infty}(I, V) \cap C^{0,1}\left(I, B_{2}^{1}(0,1)\right)$;

(ii) $u$ has (a.e. in $I)$ a strong derivative $d u / d t \in L^{\infty}\left(I, B_{2}^{1}(0,1)\right)$;

(iii) $u(0)=U_{0}$ in $B_{2}^{1}(0,1)$;

(iv) the identity

$$
\left(\frac{d u}{d t}(t), \phi\right)_{B_{2}^{1}}+(u(t), \phi)=(f(t, u(t)), \phi)_{B_{2}^{1}}
$$

takes place for all $\phi \in V$ and a.e. $t \in I$.

We remark that the fulfillment of the integral condition (1.11) is included in the fact that $u(t) \in V$ for a.e. $t \in I$.

To close this section, we announce the main result of the paper.

Theorem 2.3. Under assumptions (H1)-(H3), problem (1.8)-(1.11) admits a unique weak solution $u$ in the sense of Definition 2.2 that depends continuously on the right-hand side $f$ and the initial function $U_{0}$. Moreover, the following convergence properties hold:

$$
\begin{gathered}
u^{(n)} \longrightarrow u \quad \text { in } C\left(I, B_{2}^{1}(0,1)\right), \\
u^{(n)}(t) \longrightarrow u(t) \quad \text { in } V, \forall t \in I, \\
\frac{d u^{(n)}}{d t} \longrightarrow \frac{d u}{d t} \quad \text { in } L^{2}\left(I, B_{2}^{1}(0,1)\right),
\end{gathered}
$$

as $n$ tends to infinity, where $\left\{u^{(n)}\right\}_{n}$ is the sequence of Rothe approximations defined in (3.7).

The proof of this result will be carried out along the following sections. 
6 A semilinear heat equation with a nonlocal condition

\section{Rothe approximations}

Let $n$ be a positive integer. Following the idea of Rothe, we solve the recurrent system of time-discretized problems:

$$
\begin{gathered}
\delta u_{j}-\frac{d^{2} u_{j}}{d x^{2}}=f_{j}, \quad x \in(0,1), \\
\frac{d u_{j}}{d x}(0)=0, \\
\int_{0}^{1} u_{j}(x) d x=0,
\end{gathered}
$$

successively for $j=1, \ldots, n$, commencing with the initial value $u_{0}=U_{0}$, where $t_{j}=j h$, $h=T / n$, and

$$
\begin{gathered}
\delta u_{j}:=\frac{u_{j}-u_{j-1}}{h}, \\
f_{j}(x):=f\left(x, t_{j}, u_{j-1}\right) .
\end{gathered}
$$

For the functions $u_{j}$ which can be viewed as backward finite difference approximations of $u\left(t_{j}, \cdot\right)$, we have the following result.

Theorem 3.1. For all $n \geqslant 1$ and for all $j=1, \ldots, n$, problem $(3.1)_{j}-(3.3)_{j}$ possesses a unique solution $u_{j}$ in $H^{2}(0,1)$.

Proof. Similarly as in [5], the proof consists of the following two steps.

Step 1. We first look for the functions $w_{j}(x)=w_{j}(x ; \lambda)$ which solve the associated classical Neumann boundary value problems

$$
-\frac{d^{2} w_{j}}{d x^{2}}+\frac{1}{h} w_{j}=F_{j}, \quad x \in(0,1), \quad \frac{d w_{j}}{d x}(0)=0, \quad \frac{d w_{j}}{d x}(1)=\lambda
$$

successively for $j=1, \ldots, n$, where $F_{j}(x):=f\left(x, t_{j}, w_{j-1}\right)+(1 / h) w_{j-1}(x), w_{0}=U_{0}$ and $\lambda$ is a real parameter.

Since, according to assumptions (H1) and (H2), $F_{1}:=f\left(t_{1}, U_{0}\right)+(1 / h) U_{0} \in L^{2}(0,1)$, the Lax-Milgram lemma guarantees the existence and uniqueness of a strong solution $w_{1}=w_{1}(\cdot ; \lambda) \in H^{2}(0,1)$ to the elliptic problem $(3.4)_{1}$. Then $F_{2}:=f\left(t_{2}, w_{1}\right)+(1 / h) w_{1} \in$ $L^{2}(0,1)$, so that problem $(3.4)_{2}$ admits a unique strong solution $w_{2}=w_{2}(\cdot ; \lambda) \in H^{2}(0,1)$ thanks to Lax-Milgram lemma. Step by step, each $w_{j}$ is then uniquely determined in terms of $U_{0}, w_{1}, \ldots, w_{j-1}$. Thus, for all $n \geqslant 1$ and all $\lambda \in \mathbb{R}$, the auxiliary problems $(3.4)_{j}$, $j=1, \ldots, n$, have unique solutions $w_{j} \in H^{2}(0,1)$.

Step 2. Now, let us show that for all $j=1, \ldots, n$, the parameter $\lambda$ can be selected in a suitable way such that the corresponding function $w_{j}(\cdot ; \lambda)$ is exactly a solution of problem $(3.1)_{j}-(3.3)_{j}$. Obviously, this happens if and only if $\lambda$ is a root of the real function $\Phi_{j}(\lambda)$ defined by

$$
\Phi_{j}(\lambda):=\int_{0}^{1} w_{j}(x ; \lambda) d x
$$


so that solving the equation $\Phi_{j}(\lambda)=0$ will provide all the solutions to problem $(3.1)_{j}-$ $(3.3)_{j}$. If, in particular, this equation admits a unique solution, so is problem $(3.1)_{j}-(3.3)_{j}$.

From the superposition principle, we have that

$$
w_{j}(\cdot ; \lambda)=w_{j}(\cdot ; 0)+\chi(\cdot ; \lambda)
$$

where $w_{j}(\cdot ; 0)$ is the solution (uniquely determined) to problem $(3.4)_{j}$ for $\lambda=0$ and $\chi$ is the (unique) solution to the following problem:

$$
\begin{gathered}
-\frac{d^{2} \chi}{d x^{2}}+\frac{1}{h} \chi=0, \quad x \in(0,1), \\
\frac{d \chi}{d x}(0)=0, \quad \frac{d \chi}{d x}(1)=\lambda .
\end{gathered}
$$

One can readily find that $\chi$ is given by

$$
\chi(x ; \lambda)=\lambda \sqrt{h} \frac{\cosh (x / \sqrt{h})}{\sinh (1 / \sqrt{h})}
$$

so that, replacing into $(3.5)_{j}$, this yields

$$
\Phi_{j}(\lambda)=\frac{\lambda \sqrt{h}}{\sinh (1 / \sqrt{h})} \int_{0}^{1} \cosh \left(\frac{x}{\sqrt{h}}\right) d x+\int_{0}^{1} w_{j}(x ; 0) d x,
$$

that is,

$$
\Phi_{j}(\lambda)=h \lambda+\int_{0}^{1} w_{j}(x ; 0) d x
$$

which shows that for all $h>0, \Phi_{j}$ admits a unique root $\lambda=\lambda_{j} \in \mathbb{R}$, namely $\lambda_{j}=$ $-(1 / h) \int_{0}^{1} w_{j}(x ; 0) d x$. Hence, problem $(3.1)_{j}-(3.3)_{j}$ is uniquely solvable for all $n \geqslant 1$ and all $j=1, \ldots, n$. Therefore, Theorem 3.1 has been proved.

Now, for all $n \geqslant 1$, we introduce the Rothe approximation $u^{(n)}: I \rightarrow H^{2}(0,1) \cap V$ is defined by

$$
u^{(n)}(t)=u_{j-1}+\delta u_{j}\left(t-t_{j-1}\right), \quad t \in\left[t_{j-1}, t_{j}\right], j=1, \ldots, n,
$$

and the corresponding step function $\bar{u}^{(n)}: I \rightarrow H^{2}(0,1) \cap V$ is defined as follows:

$$
\bar{u}^{(n)}(t)= \begin{cases}u_{j} & \text { for } t \in\left(t_{j-1}, t_{j}\right], j=1, \ldots, n, \\ U_{0} & \text { for } t=0\end{cases}
$$

We expect that the limit $\lim _{n \rightarrow \infty} u^{(n)}=u$ exists in a suitable sense, and that is precisely the desired weak solution to our problem (1.8)-(1.11). The establishment of this fact requires some a priori estimates whose derivation is the subject of the following section. 
8 A semilinear heat equation with a nonlocal condition

\section{A priori estimates for the approximations}

Lemma 4.1. There exist $c>0$ such that for all $n \geqslant 1$, the solutions $u_{j}$ of the time-discretized problems $(3.1)_{j}-(3.3)_{j}, j=1, \ldots, n$, obey the estimates

$$
\begin{gathered}
\left\|u_{j}\right\| \leqslant c, \\
\left\|\delta u_{j}\right\|_{B_{2}^{1}} \leqslant c .
\end{gathered}
$$

Proof. The key point to establish these estimates is the derivation of a nonstandard variational formulation of problems $(3.1)_{j}-(3.3)_{j}$. To this aim, we take, for all $j=1, \ldots, n$, the inner product in $B_{2}^{1}(0,1)$ of $(3.1) j$ with any function $\phi$ from the space $V$ defined in $(2.1)$ to get

$$
\left(\delta u_{j}, \phi\right)_{B_{2}^{1}(0,1)}-\left(\frac{d^{2} u_{j}}{d x^{2}}, \phi\right)_{B_{2}^{1}(0,1)}=\left(f_{j}, \phi\right)_{B_{2}^{1}(0,1)} .
$$

But from $(3.2)_{j}$ we have

$$
\begin{aligned}
\left(\frac{d^{2} u_{j}}{d x^{2}}, \phi\right)_{B_{2}^{1}(0,1)} & =\int_{0}^{1} \frac{d u_{j}}{d x}(x) \mathfrak{J}_{x} \phi d x \\
& =\left.u_{j}(x) \mathfrak{I}_{x} \phi\right|_{x=0} ^{x=1}-\int_{0}^{1} u_{j} \phi d x
\end{aligned}
$$

then

$$
\left(\frac{d^{2} u_{j}}{d x^{2}}, \phi\right)_{B_{2}^{1}(0,1)}=-\left(u_{j}, \phi\right),
$$

since $\phi \in V$. Substituting in (4.3), this yields the required variational form:

$$
\left(\delta u_{j}, \phi\right)_{B_{2}^{1}(0,1)}+\left(u_{j}, \phi\right)=\left(f_{j}, \phi\right)_{B_{2}^{1}(0,1)},
$$

which gives for $j=1$ that

$$
\left(\delta u_{1}, \phi\right)_{B_{2}^{1}(0,1)}+h\left(\delta u_{1}, \phi\right)=\left(f_{1}, \phi\right)_{B_{2}^{1}(0,1)}-\left(U_{0}, \phi\right), \quad \forall \phi \in V .
$$

Integrating by parts the second term in the right-hand side of (4.6), we have

$$
\begin{aligned}
\left(U_{0}, \phi\right) & =\int_{0}^{1} U_{0}(x) \frac{d}{d x}\left(\mathfrak{I}_{x} \phi\right) d x \\
& =\left.U_{0}(x) \mathfrak{I}_{x} \phi\right|_{x=0} ^{x=1}-\int_{0}^{1} \frac{d U_{0}}{d x}(x) \mathfrak{I}_{x} \phi d x \\
& =-\int_{0}^{1} \frac{d U_{0}}{d x}(x) \mathfrak{I}_{x} \phi d x,
\end{aligned}
$$

but, due to assumption $(\mathrm{H} 3)_{1}$, we note that

$$
\mathfrak{I}_{x}\left(\frac{d^{2} U_{0}}{d x^{2}}(x)\right)=\frac{d U_{0}}{d x}(x) \quad \forall x \in(0,1),
$$


whence

$$
\left(U_{0}, \phi\right)=-\int_{0}^{1} \mathfrak{J}_{x}\left(\frac{d^{2} U_{0}}{d x^{2}}(x)\right) \mathfrak{J}_{x} \phi d x
$$

so that (4.6) becomes

$$
\left(\delta u_{1}, \phi\right)_{B_{2}^{1}(0,1)}+h\left(\delta u_{1}, \phi\right)=\left(f_{1}+\frac{d^{2} U_{0}}{d x^{2}}, \phi\right)_{B_{2}^{1}(0,1)}, \quad \forall \phi \in V .
$$

Testing this last equality with $\phi=\delta u_{1}=\left(u_{1}-U_{0}\right) / h$ which is clearly an element of $V$ because of $(3.3)_{1}$ and assumption $(\mathrm{H} 3)_{2}$, we derive with the help of Cauchy-Schwarz inequality

$$
\left\|\delta u_{1}\right\|_{B_{2}^{1}}^{2}+h\left\|\delta u_{1}\right\|^{2} \leqslant\left[\left\|f_{1}\right\|_{B_{2}^{1}}+\left\|\frac{d^{2} U_{0}}{d x^{2}}\right\|_{B_{2}^{1}}\right]\left\|\delta u_{1}\right\|_{B_{2}^{1}},
$$

consequently

$$
\left\|\delta u_{1}\right\|_{B_{2}^{1}} \leqslant\left\|f\left(t_{1}, U_{0}\right)\right\|_{B_{2}^{1}}+\left\|\frac{d^{2} U_{0}}{d x^{2}}\right\|_{B_{2}^{1}}
$$

and then

$$
\left\|\delta u_{1}\right\|_{B_{2}^{1}} \leqslant \max _{t \in I}\left\|f\left(t, U_{0}\right)\right\|_{B_{2}^{1}}+\left\|\frac{d^{2} U_{0}}{d x^{2}}\right\|_{B_{2}^{1}}:=c_{1} .
$$

Next, subtracting $(4.4)_{j-1}$ from $(4.4)_{j}(j=2, \ldots, n)$ and putting $\phi=\delta u_{j}$ which belongs to $V$ in view of $(3.3)_{j-1}$ and $(3.3)_{j}$, we estimate

$$
\left\|\delta u_{j}\right\|_{B_{2}^{1}}^{2}+\frac{1}{h}\left\|u_{j}-u_{j-1}\right\|^{2} \leqslant\left(\left\|f_{j}-f_{j-1}\right\|_{B_{2}^{1}}+\left\|\delta u_{j-1}\right\|_{B_{2}^{1}}\right)\left\|\delta u_{j}\right\|_{B_{2}^{1}}
$$

which implies that

$$
\left\|\delta u_{j}\right\|_{B_{2}^{1}} \leqslant\left\|f_{j}-f_{j-1}\right\|_{B_{2}^{1}}+\left\|\delta u_{j-1}\right\|_{B_{2}^{1}}
$$

then, iterating we may arrive at

$$
\left\|\delta u_{j}\right\|_{B_{2}^{1}} \leqslant \sum_{i=2}^{j}\left\|f_{i}-f_{i-1}\right\|_{B_{2}^{1}}+\left\|\delta u_{1}\right\|_{B_{2}^{1}} .
$$

But owing to assumption ( $\mathrm{H} 1)$, we have for all $i \geqslant 2$ that

$$
\begin{aligned}
\left\|f_{i}-f_{i-1}\right\|_{B_{2}^{1}} & =\left\|f\left(t_{i}, u_{i-1}\right)-f\left(t_{i-1}, u_{i-2}\right)\right\|_{B_{2}^{1}} \\
& \leqslant l\left[h\left(1+\left\|u_{i-1}\right\|_{B_{2}^{1}}+\left\|u_{i-2}\right\|_{B_{2}^{1}}\right)+\left\|u_{i-1}-u_{i-2}\right\|_{B_{2}^{1}}\right] \\
& =\operatorname{lh}\left[1+\left\|u_{i-1}\right\|_{B_{2}^{1}}+\left\|u_{i-2}\right\|_{B_{2}^{1}}+\left\|\delta u_{i-1}\right\|_{B_{2}^{1}}\right],
\end{aligned}
$$


10 A semilinear heat equation with a nonlocal condition

so that

$$
\begin{aligned}
\sum_{i=2}^{j}\left\|f_{i}-f_{i-1}\right\|_{B_{2}^{1}} & \leqslant \operatorname{lh}\left[(j-1)+\sum_{i=1}^{j-1}\left\|u_{i}\right\|_{B_{2}^{1}}+\sum_{i=0}^{j-2}\left\|u_{i}\right\|_{B_{2}^{1}}+\sum_{i=1}^{j-1}\left\|\delta u_{i}\right\|_{B_{2}^{1}}\right] \\
& \leqslant l(j-1) h+2 \operatorname{lh} \sum_{i=1}^{j-1}\left\|u_{i}\right\|_{B_{2}^{1}}+\operatorname{lh}\left\|U_{0}\right\|_{B_{2}^{1}}+\operatorname{lh} \sum_{i=1}^{j-1}\left\|\delta u_{i}\right\|_{B_{2}^{1}} .
\end{aligned}
$$

To dominate the right-hand side in (4.18), we need to estimate the term $\left\|u_{i}\right\|_{B_{2}^{1}}$. For this, we take $\phi=u_{i}$ in $(4.4)_{i}, i=1, \ldots, n$, and get

$$
\frac{1}{h}\left\|u_{i}\right\|_{B_{2}^{1}}^{2}+\left\|u_{i}\right\|^{2} \leqslant\left(\left\|f_{i}\right\|_{B_{2}^{1}}+\frac{1}{h}\left\|u_{i-1}\right\|_{B_{2}^{1}}\right)\left\|u_{i}\right\|_{B_{2}^{1}}
$$

from where we derive

$$
\begin{aligned}
\left\|u_{i}\right\|_{B_{2}^{1}} & \leqslant h\left\|f_{i}\right\|_{B_{2}^{1}}+\left\|u_{i-1}\right\|_{B_{2}^{1}} \\
& \leqslant h\left(\left\|f_{i}\right\|_{B_{2}^{1}}+\left\|f_{i-1}\right\|_{B_{2}^{1}}\right)+\left\|u_{i-2}\right\|_{B_{2}^{1}},
\end{aligned}
$$

and from this recurrent inequality, we successively estimate

$$
\left\|u_{i}\right\|_{B_{2}^{1}} \leqslant h \sum_{k=1}^{i}\left\|f_{k}\right\|_{B_{2}^{1}}+\left\|U_{0}\right\|_{B_{2}^{1}} .
$$

Invoking assumption (H1), we have for all $k \geqslant 1$ that

$$
\begin{aligned}
\left\|f_{k}\right\|_{B_{2}^{1}} & \leqslant\left\|f\left(t_{k}, u_{k-1}\right)-f\left(t_{k}, 0\right)\right\|_{B_{2}^{1}}+\left\|f\left(t_{k}, 0\right)\right\|_{B_{2}^{1}} \\
& \leqslant l\left\|u_{k-1}\right\|_{B_{2}^{1}}+M,
\end{aligned}
$$

where $M:=\max _{t \in I}\|f(t, 0)\|_{B_{2}^{1}}<+\infty$. Substituting (4.22) in the previous inequality, we get

$$
\begin{aligned}
\left\|u_{i}\right\|_{B_{2}^{1}} & \leqslant h \sum_{k=1}^{i}\left(l\left\|u_{k-1}\right\|_{B_{2}^{1}}+M\right)+\left\|U_{0}\right\|_{B_{2}^{1}} \\
& =i h M+(1+l h)\left\|U_{0}\right\|_{B_{2}^{1}}+l h \sum_{k=2}^{i}\left\|u_{k-1}\right\|_{B_{2}^{1}} \\
& \leqslant T M+(1+l h)\left\|U_{0}\right\|_{B_{2}^{1}}+l h \sum_{k=1}^{i-1}\left\|u_{k}\right\|_{B_{2}^{1}},
\end{aligned}
$$

from where it comes due to the discrete Gronwall's lemma that

$$
\left\|u_{i}\right\|_{B_{2}^{1}} \leqslant\left(T M+(1+l h)\left\|U_{0}\right\|_{B_{2}^{1}}\right) e^{l(i-1) h},
$$

hence

$$
\left\|u_{i}\right\|_{B_{2}^{1}} \leqslant\left(T M+(1+l T)\left\|U_{0}\right\|_{B_{2}^{1}}\right) e^{l T}:=c_{2} .
$$


Now, returning to (4.18), we can write thanks to (4.25) that

$$
\begin{aligned}
\sum_{i=2}^{j}\left\|f_{i}-f_{i-1}\right\|_{B_{2}^{1}} & \leqslant l(j-1) h+l h\left(2(j-1) c_{2}+\left\|U_{0}\right\|_{B_{2}^{1}}\right)+l h \sum_{i=1}^{j-1}\left\|\delta u_{i}\right\|_{B_{2}^{1}} \\
& \leqslant l T\left(1+2 c_{2}+\left\|U_{0}\right\|_{B_{2}^{1}}\right)+l h \sum_{i=1}^{j-1}\left\|\delta u_{i}\right\|_{B_{2}^{1}} .
\end{aligned}
$$

Combining (4.13), (4.16), and the last inequality, we have

$$
\left\|\delta u_{j}\right\|_{B_{2}^{1}} \leqslant c_{1}+l T\left(1+2 c_{2}+\left\|U_{0}\right\|_{B_{2}^{1}}\right)+l h \sum_{i=1}^{j-1}\left\|\delta u_{i}\right\|_{B_{2}^{1}},
$$

hence, applying Gronwall's lemma in discrete form again, we get

$$
\left\|\delta u_{j}\right\|_{B_{2}^{1}} \leqslant\left[c_{1}+l T\left(1+2 c_{2}+\left\|U_{0}\right\|_{B_{2}^{1}}\right)\right] e^{l(j-1) h} .
$$

Thus, estimate (4.2) is proved for $c=c_{3}$ with

$$
c_{3}:=\left[c_{1}+l T\left(1+2 c_{2}+\left\|U_{0}\right\|_{B_{2}^{1}}\right)\right] e^{l T} .
$$

Next, to derive estimate (4.1), we insert $\phi=u_{j}-u_{j-1}$ in $(4.4)_{j}$ and apply the identity

$$
\left(u_{j}, u_{j}-u_{j-1}\right)=\frac{1}{2}\left(\left\|u_{j}-u_{j-1}\right\|^{2}+\left\|u_{j}\right\|^{2}-\left\|u_{j-1}\right\|^{2}\right)
$$

to get

$$
h\left\|\delta u_{j}\right\|_{B_{2}^{1}}^{2}+\frac{1}{2}\left\|u_{j}-u_{j-1}\right\|^{2}+\frac{1}{2}\left\|u_{j}\right\|^{2}=\left(f_{j}, u_{j}-u_{j-1}\right)_{B_{2}^{1}}+\frac{1}{2}\left\|u_{j-1}\right\|^{2} .
$$

Ignoring the first two terms in the left-hand side, we obtain

$$
\left\|u_{j}\right\|^{2} \leqslant 2\left\|f_{j}\right\|_{B_{2}^{1}}\left\|u_{j}-u_{j-1}\right\|_{B_{2}^{1}}+\left\|u_{j-1}\right\|^{2}
$$

whence, using (4.22), (4.25), and (4.2),

$$
\left\|u_{j}\right\|^{2} \leqslant 2 h\left(l_{c_{2}}+M\right) c_{3}+\left\|u_{j-1}\right\|^{2} .
$$

So, by an iterative procedure, we get

$$
\left\|u_{j}\right\|^{2} \leqslant 2 j h\left(l c_{2}+M\right) c_{3}+\left\|U_{0}\right\|^{2}
$$

from where estimate (4.1) follows with $c=c_{4}$, where

$$
c_{4}:=\left\{2 T\left(l c_{2}+M\right) c_{3}+\left\|U_{0}\right\|^{2}\right\}^{1 / 2},
$$

and so the proof is complete. 
12 A semilinear heat equation with a nonlocal condition

If we extend, for all $n \geqslant 1$, the function $\bar{u}^{(n)}$ defined on $I$ to the interval $[-T / n, 0)$ by setting

$$
\bar{u}^{(n)}(t)=U_{0}, \quad \forall t \in\left[-\frac{T}{n}, 0\right),
$$

we can state the following corollary.

Corollary 4.2. For all $n \geqslant 1$, the functions $u^{(n)}$ and $\bar{u}^{(n)}$ satisfy the estimates

$$
\begin{gathered}
\left\|u^{(n)}(t)\right\| \leqslant c, \quad\left\|\bar{u}^{(n)}(t)\right\| \leqslant c, \quad \forall t \in I, \\
\left\|\frac{d u^{(n)}}{d t}(t)\right\|_{B_{2}^{1}} \leqslant c, \quad \text { a.e. in } I, \\
\left\|\bar{u}^{(n)}(t)-u^{(n)}(t)\right\|_{B_{2}^{1}} \leqslant \frac{c}{n}, \quad \forall t \in I, \\
\left\|\bar{u}^{(n)}(t)-\bar{u}^{(n)}\left(t-\frac{T}{n}\right)\right\|_{B_{2}^{1}} \leqslant \frac{c}{n}, \quad \forall t \in I .
\end{gathered}
$$

Proof. Both estimates (4.37) follow immediately from (4.1) with the same constant $c=c_{4}$. On the other hand, invoking the identity

$$
\frac{d u^{(n)}}{d t}(t)=\delta u_{j}, \quad \forall t \in\left(t_{j-1}, t_{j}\right], 1 \leqslant j \leqslant n,
$$

estimate (4.38) is seen to be an easy consequence of estimate (4.2) with $c=c_{3}$. Next, observing that we have

$$
\begin{gathered}
\bar{u}^{(n)}(t)-u^{(n)}(t)= \begin{cases}\left(t_{j}-t\right) \delta u_{j}, & \forall t \in\left(t_{j-1}, t_{j}\right], 1 \leqslant j \leqslant n, \\
0, & t=0,\end{cases} \\
\bar{u}^{(n)}(t)-\bar{u}^{(n)}\left(t-\frac{T}{n}\right)= \begin{cases}u_{j}-u_{j-1}, & \forall t \in\left(t_{j-1}, t_{j}\right], 1 \leqslant j \leqslant n, \\
0, & t=0,\end{cases}
\end{gathered}
$$

we can write

$$
\begin{gathered}
\left\|\bar{u}^{(n)}(t)-u^{(n)}(t)\right\|_{B_{2}^{1}} \leqslant h \max _{1 \leqslant j \leqslant n}\left\|\delta u_{j}\right\|_{B_{2}^{1}} \quad \forall t \in I, \\
\left\|\bar{u}^{(n)}(t)-\bar{u}^{(n)}\left(t-\frac{T}{n}\right)\right\|_{B_{2}^{1}} \leqslant h \max _{1 \leqslant j \leqslant n}\left\|\delta u_{j}\right\|_{B_{2}^{1}}, \quad \forall t \in I,
\end{gathered}
$$

hence, in view of (4.2), we get the required estimates (4.39) and (4.40) with $c=c_{3} T$.

\section{Existence, uniqueness, and convergence of the method}

Let us define, for all $n \geqslant 1$, the abstract step function $\bar{f}^{(n)}: I \times V \rightarrow L^{2}(0,1)$ by

$$
\bar{f}^{(n)}(t, v)=f\left(t_{j}, v\right), \quad \forall t \in\left(t_{j-1}, t_{j}\right], \quad j=1, \ldots, n .
$$


Then, the variational equations $(4.4)_{j}$ may be rewritten in the form

$$
\left(\frac{d u^{(n)}}{d t}(t), \phi\right)_{B_{2}^{1}}+\left(\bar{u}^{(n)}(t), \phi\right)=\left(\bar{f}^{(n)}\left(t, \bar{u}^{(n)}\left(t-\frac{T}{n}\right)\right), \phi\right)_{B_{2}^{1}},
$$

for all $\phi \in V$ and $t \in(0, T]$.

It is convenient to present now a basic convergence statement.

Theorem 5.1. The sequence $\left\{u^{(n)}\right\}_{n}$ converges in the norm of the space $C\left(I, B_{2}^{1}(0,1)\right)$ to some function $u \in C\left(I, B_{2}^{1}(0,1)\right)$ and the error estimate

$$
\left\|u^{(n)}-u\right\|_{C\left(I, B_{2}^{1}(0,1)\right)} \leqslant \frac{c}{n^{1 / 2}}
$$

holds for all $n \geqslant 1$.

Proof. The idea of the proof consists in showing that $\left\{u^{(n)}\right\}_{n}$ is a Cauchy sequence in the Banach space $C\left(I, B_{2}^{1}(0,1)\right)$.

Let $u^{(n)}$ and $u^{(m)}$ be the Rothe approximations corresponding to the step lengths $h_{n}=$ $T / n$ and $h_{m}=T / m$, respectively, with $m>n \geqslant 1$. Take the difference $(5.1)^{n}-(5.1)^{m}$ tested with $\phi=u^{(n)}(t)-u^{(m)}(t)(\in V)$, this yields for all $t \in(0, T]$ that

$$
\begin{gathered}
\left(\frac{d}{d t}\left(u^{(n)}(t)-u^{(m)}(t)\right), u^{(n)}(t)-u^{(m)}(t)\right)_{B_{2}^{1}}+\left(\bar{u}^{(n)}(t)-\bar{u}^{(m)}(t), u^{(n)}(t)-u^{(m)}(t)\right) \\
=\left(\bar{f}^{(n)}\left(t, \bar{u}^{(n)}\left(t-\frac{T}{n}\right)\right)-\bar{f}^{(m)}\left(t, \bar{u}^{(m)}\left(t-\frac{T}{m}\right)\right), u^{(n)}(t)-u^{(m)}(t)\right)_{B_{2}^{1}}
\end{gathered}
$$

or after some rearrangement,

$$
\begin{aligned}
\frac{1}{2} \frac{d}{d t}\left\|u^{(n)}(t)-u^{(m)}(t)\right\|_{B_{2}^{1}}^{2}+\left\|\bar{u}^{(n)}(t)-\bar{u}^{(m)}(t)\right\|^{2} \\
=\left(\bar{u}^{(n)}(t)-\bar{u}^{(m)}(t), \bar{u}^{(n)}(t)-\bar{u}^{(m)}(t)-u^{(n)}(t)+u^{(m)}(t)\right) \\
\quad+\left(\bar{f}^{(n)}\left(t, \bar{u}^{(n)}\left(t-\frac{T}{n}\right)\right)-\bar{f}^{(m)}\left(t, \bar{u}^{(m)}\left(t-\frac{T}{m}\right)\right), u^{(n)}(t)-u^{(m)}(t)\right)_{B_{2}^{1}} .
\end{aligned}
$$

But since we have

$$
\bar{f}^{(n)}\left(t, \bar{u}^{(n)}\left(t-\frac{T}{n}\right)\right)=f\left(t_{j}, u_{j-1}\right):=f_{j}, \quad \forall t \in\left(t_{j-1}, t_{j}\right], j=1, \ldots, n,
$$

it follows in view of (4.22) that

$$
\begin{aligned}
\left\|\bar{f}^{(n)}\left(t, \bar{u}^{(n)}\left(t-\frac{T}{n}\right)\right)\right\|_{B_{2}^{1}} & \leqslant \max _{1 \leqslant j \leqslant n}\left\|f_{j}\right\|_{B_{2}^{1}} \\
& \leqslant l \max _{1 \leqslant j \leqslant n}\left\|u_{j-1}\right\|_{B_{2}^{1}}+M, \quad \forall t \in(0, T],
\end{aligned}
$$


14 A semilinear heat equation with a nonlocal condition

hence due to (4.25),

$$
\left\|\bar{f}^{(n)}\left(t, \bar{u}^{(n)}\left(t-\frac{T}{n}\right)\right)\right\|_{B_{2}^{1}} \leqslant l c_{2}+M, \quad \forall t \in(0, T] .
$$

Thus, estimating the identity

$$
\left(\bar{u}^{(n)}(t), \phi\right)=\left(\bar{f}^{(n)}\left(t, \bar{u}^{(n)}\left(t-\frac{T}{n}\right)\right)-\frac{d u^{(n)}}{d t}(t), \phi\right)_{B_{2}^{1}}, \quad \forall t \in(0, T], \forall \phi \in V,
$$

which follows from $(5.1)^{n}$, we obtain owing to (4.38) that

$$
\begin{aligned}
\left|\left(\bar{u}^{(n)}(t), \phi\right)\right| & \leqslant\left[\left\|\bar{f}^{(n)}\left(t, \bar{u}^{(n)}\left(t-\frac{T}{n}\right)\right)\right\|_{B_{2}^{1}}+\left\|\frac{d u^{(n)}}{d t}(t)\right\|_{B_{2}^{1}}\right]\|\phi\|_{B_{2}^{1}} \\
& \leqslant c_{5}\|\phi\|_{B_{2}^{1}}, \quad \forall t \in(0, T],
\end{aligned}
$$

with $c_{5}:=l c_{2}+M+c_{3}$. This, together with (4.39), allows us to majorize the first term in the right-hand side of (5.4) as follows:

$$
\begin{aligned}
& \left(\bar{u}^{(n)}(t)-\bar{u}^{(m)}(t), \bar{u}^{(n)}(t)-\bar{u}^{(m)}(t)-u^{(n)}(t)+u^{(m)}(t)\right) \\
& \quad \leqslant 2 c_{5}\left(\left\|\bar{u}^{(n)}(t)-u^{(n)}(t)\right\|_{B_{2}^{1}}+\left\|\bar{u}^{(m)}(t)-u^{(m)}(t)\right\|_{B_{2}^{1}}\right) \\
& \quad \leqslant c_{6}\left(\frac{1}{n}+\frac{1}{m}\right), \quad \forall t \in(0, T],
\end{aligned}
$$

with $c_{6}:=2 c_{5} c_{3} T$.

On the other hand, thanks to the Cauchy inequality (2.12), we can write for every $\varepsilon>0$ that

$$
\begin{aligned}
&\left(\bar{f}^{(n)}\left(t, \bar{u}^{(n)}\left(t-\frac{T}{n}\right)\right)-\bar{f}^{(m)}\left(t, \bar{u}^{(m)}\left(t-\frac{T}{m}\right)\right), u^{(n)}(t)-u^{(m)}(t)\right)_{B_{2}^{1}} \\
& \leqslant\left\|\bar{f}^{(n)}\left(t, \bar{u}^{(n)}\left(t-\frac{T}{n}\right)\right)-\bar{f}^{(m)}\left(t, \bar{u}^{(m)}\left(t-\frac{T}{m}\right)\right)\right\|\left\|_{B_{2}^{1}}\right\| u^{(n)}(t)-u^{(m)}(t) \|_{B_{2}^{1}} \\
& \leqslant \frac{\varepsilon}{2}\left\|\bar{f}^{(n)}\left(t, \bar{u}^{(n)}\left(t-\frac{T}{n}\right)\right)-\bar{f}^{(m)}\left(t, \bar{u}^{(m)}\left(t-\frac{T}{m}\right)\right)\right\|_{B_{2}^{1}}^{2} \\
&+\frac{1}{2 \varepsilon}\left\|u^{(n)}(t)-u^{(m)}(t)\right\|_{B_{2}^{1}}^{2}, \quad \forall t \in(0, T] .
\end{aligned}
$$

Now, let $t$ be arbitrary but fixed in $(0, T]$, then there exist two integers $k$ and $i$ corresponding to the subdivision of $I$ into $n$ and $m$ subintervals, respectively, such that 
$t \in\left(t_{k-1}, t_{k}\right] \cap\left(t_{i-1}, t_{i}\right]$, hence from assumption (H1), it follows that

$$
\begin{gathered}
\left\|\bar{f}^{(n)}\left(t, \bar{u}^{(n)}\left(t-\frac{T}{n}\right)\right)-\bar{f}^{(m)}\left(t, \bar{u}^{(m)}\left(t-\frac{T}{m}\right)\right)\right\|_{B_{2}^{1}}^{2} \\
=\left\|f\left(t_{k}, \bar{u}^{(n)}\left(t-\frac{T}{n}\right)\right)-f\left(t_{i}, \bar{u}^{(m)}\left(t-\frac{T}{m}\right)\right)\right\|_{B_{2}^{1}}^{2} \\
\leqslant l^{2}\left[\left|t_{k}-t_{i}\right|\left\{1+\left\|\bar{u}^{(n)}\left(t-\frac{T}{n}\right)\right\|_{B_{2}^{1}}+\left\|\bar{u}^{(m)}\left(t-\frac{T}{m}\right)\right\|_{B_{2}^{1}}\right\}\right. \\
\left.\left.\quad+\left\|\bar{u}^{(n)}\left(t-\frac{T}{n}\right)-\bar{u}^{(m)}\left(t-\frac{T}{m}\right)\right\|_{B_{2}^{1}}\right]\right]^{2} \\
\leqslant l^{2}\left[\left(h_{n}+h_{m}\right)\left(1+\left\|u_{k-1}\right\|_{B_{2}^{1}}+\left\|u_{i-1}\right\|_{B_{2}^{1}}\right)+\left\|\bar{u}^{(n)}\left(t-\frac{T}{n}\right)-\bar{u}^{(n)}(t)\right\|_{B_{2}^{1}}\right. \\
\left.\quad+\left\|\bar{u}^{(n)}(t)-\bar{u}^{(m)}(t)\right\|_{B_{2}^{1}}+\left\|\bar{u}^{(m)}(t)-\bar{u}^{(m)}\left(t-\frac{T}{m}\right)\right\|_{B_{2}^{1}}\right]^{2},
\end{gathered}
$$

consequently, with consideration to (4.25) and (4.40), we deduce that

$$
\begin{aligned}
& \left\|\bar{f}^{(n)}\left(t, \bar{u}^{(n)}\left(t-\frac{T}{n}\right)\right)-\bar{f}^{(m)}\left(t, \bar{u}^{(m)}\left(t-\frac{T}{m}\right)\right)\right\|_{B_{2}^{1}}^{2} \\
& \leqslant l^{2}\left[T\left(\frac{1}{n}+\frac{1}{m}\right)\left(1+2 c_{2}\right)+c_{3} T\left(\frac{1}{n}+\frac{1}{m}\right)+\left\|\bar{u}^{(n)}(t)-\bar{u}^{(m)}(t)\right\|_{B_{2}^{1}}\right]^{2} \\
& =l^{2}\left[T\left(1+2 c_{2}+c_{3}\right)\left(\frac{1}{n}+\frac{1}{m}\right)+\left\|\bar{u}^{(n)}(t)-\bar{u}^{(m)}(t)\right\|_{B_{2}^{1}}\right]^{2} \\
& \leqslant l^{2}\left[c_{7}^{2}\left(\frac{1}{n}+\frac{1}{m}\right)^{2}+2 c_{7}\left(\frac{1}{n}+\frac{1}{m}\right)\left(\left\|\bar{u}^{(n)}(t)\right\|_{B_{2}^{1}}+\left\|\bar{u}^{(m)}(t)\right\|_{B_{2}^{1}}\right)\right. \\
& \left.\quad+\left\|\bar{u}^{(n)}(t)-\bar{u}^{(m)}(t)\right\|_{B_{2}^{1}}^{2}\right] \\
& \leqslant\left(l c_{7}\right)^{2}\left(\frac{1}{n}+\frac{1}{m}\right)^{2}+4 l^{2} c_{7} c_{2}\left(\frac{1}{n}+\frac{1}{m}\right)+l^{2}\left\|\bar{u}^{(n)}(t)-\bar{u}^{(m)}(t)\right\|_{B_{2}^{1}}^{2}
\end{aligned}
$$

for all $t \in(0, T]$, with $c_{7}:=T\left(1+2 c_{2}+c_{3}\right)$. Thus, using the notations $c_{8}:=\left(l c_{7}\right)^{2}$ and $c_{9}:=4 l^{2} c_{7} c_{2}$, we write

$$
\begin{aligned}
& \left\|\bar{f}^{(n)}\left(t, \bar{u}^{(n)}\left(t-\frac{T}{n}\right)\right)-\bar{f}^{(m)}\left(t, \bar{u}^{(m)}\left(t-\frac{T}{m}\right)\right)\right\|_{B_{2}^{1}}^{2} \\
& \quad \leqslant c_{8}\left(\frac{1}{n}+\frac{1}{m}\right)^{2}+c_{9}\left(\frac{1}{n}+\frac{1}{m}\right)+l^{2}\left\|\bar{u}^{(n)}(t)-\bar{u}^{(m)}(t)\right\|_{B_{2}^{1}}^{2}, \quad \forall t \in(0, T],
\end{aligned}
$$


hence, going back to (5.11), we have

$$
\begin{aligned}
&\left(\bar{f}^{(n)}\left(t, \bar{u}^{(n)}\left(t-\frac{T}{n}\right)\right)-\bar{f}^{(m)}\left(t, \bar{u}^{(m)}\left(t-\frac{T}{m}\right)\right), u^{(n)}(t)-u^{(m)}(t)\right)_{B_{2}^{1}} \\
& \leqslant \frac{\varepsilon}{2} c_{8}\left(\frac{1}{n}+\frac{1}{m}\right)^{2}+\frac{\varepsilon}{2} c_{9}\left(\frac{1}{n}+\frac{1}{m}\right)+\frac{\varepsilon}{2} l^{2}\left\|\bar{u}^{(n)}(t)-\bar{u}^{(m)}(t)\right\|_{B_{2}^{1}}^{2} \\
&+\frac{1}{2 \varepsilon}\left\|u^{(n)}(t)-u^{(m)}(t)\right\|_{B_{2}^{1}}^{2}, \quad \forall t \in(0, T] .
\end{aligned}
$$

Next, combining (5.4), (5.10), and (5.15), we have for all $t \in(0, T]$ that

$$
\begin{aligned}
\frac{d}{d t}\left\|u^{(n)}(t)-u^{(m)}(t)\right\|_{B_{2}^{1}}^{2}+2\left\|\bar{u}^{(n)}(t)-\bar{u}^{(m)}(t)\right\|^{2} & \\
\leqslant & \varepsilon c_{8}\left(\frac{1}{n}+\frac{1}{m}\right)^{2}+\left(\varepsilon c_{9}+2 c_{6}\right)\left(\frac{1}{n}+\frac{1}{m}\right)+\varepsilon l^{2}\left\|\bar{u}^{(n)}(t)-\bar{u}^{(m)}(t)\right\|_{B_{2}^{1}}^{2} \\
& +\frac{1}{\varepsilon}\left\|u^{(n)}(t)-u^{(m)}(t)\right\|_{B_{2}^{1}}^{2},
\end{aligned}
$$

or

$$
\begin{aligned}
& \frac{d}{d t}\left\|u^{(n)}(t)-u^{(m)}(t)\right\|_{B_{2}^{1}}^{2}+\left(2-\varepsilon l^{2}\right)\left\|\bar{u}^{(n)}(t)-\bar{u}^{(m)}(t)\right\|^{2} \\
& \quad \leqslant \varepsilon c_{8}\left(\frac{1}{n}+\frac{1}{m}\right)^{2}+\left(\varepsilon c_{9}+2 c_{6}\right)\left(\frac{1}{n}+\frac{1}{m}\right)+\frac{1}{\varepsilon}\left\|u^{(n)}(t)-u^{(m)}(t)\right\|_{B_{2}^{1}}^{2} .
\end{aligned}
$$

Let us choose $\varepsilon$ so that $2-\varepsilon l^{2}=0$, that is, $\varepsilon=2 / l^{2}$ and integrate the last inequality over $(0, t)$. Then, invoking the fact that $u^{(n)}(0)=u^{(m)}(0)=U_{0}$, we obtain for all $t \in I$ that

$$
\begin{aligned}
\left\|u^{(n)}(t)-u^{(m)}(t)\right\|_{B_{2}^{1}}^{2} \leqslant & \frac{2 c_{8} T}{l^{2}}\left(\frac{1}{n}+\frac{1}{m}\right)^{2}+2 T\left(\frac{c_{9}}{l^{2}}+c_{6}\right)\left(\frac{1}{n}+\frac{1}{m}\right) \\
& +\frac{l^{2}}{2} \int_{0}^{t}\left\|u^{(n)}(\tau)-u^{(m)}(\tau)\right\|_{B_{2}^{1}}^{2} d \tau,
\end{aligned}
$$

giving by Gronwall's lemma

$$
\left\|u^{(n)}(t)-u^{(m)}(t)\right\|_{B_{2}^{1}}^{2} \leqslant\left[c_{10}\left(\frac{1}{n}+\frac{1}{m}\right)^{2}+c_{11}\left(\frac{1}{n}+\frac{1}{m}\right)\right] e^{l^{2} t / 2} \quad \forall t \in I,
$$

with $c_{10}:=2 c_{8} T / l^{2}$ and $c_{11}:=2 T\left(c_{9} / l^{2}+c_{6}\right)$. Accordingly,

$$
\left\|u^{(n)}(t)-u^{(m)}(t)\right\|_{B_{2}^{1}} \leqslant\left[c_{10}\left(\frac{1}{n}+\frac{1}{m}\right)^{2}+c_{11}\left(\frac{1}{n}+\frac{1}{m}\right)\right]^{1 / 2} e^{l^{2} T / 4}, \quad \forall t \in I,
$$

then, taking the supremum with respect to $t$ in the left-hand side of this inequality, we have

$$
\left\|u^{(n)}-u^{(m)}\right\|_{C\left(I, B_{2}^{1}(0,1)\right)} \leqslant\left[c_{10}\left(\frac{1}{n}+\frac{1}{m}\right)^{2}+c_{11}\left(\frac{1}{n}+\frac{1}{m}\right)\right]^{1 / 2} e^{l^{2} T / 4}
$$


which implies the existence of a function $u \in C\left(I, B_{2}^{1}(0,1)\right)$ such that $u^{(n)} \rightarrow u$ in this space. Moreover, passing to the limit $m \rightarrow \infty$ in (5.21), we obtain the error estimate (5.2) with $c=c_{12}:=\sqrt{c_{10}+c_{11}} e^{l^{2} T / 4}$, which achieves the proof.

Next, some properties of the function $u$ from Theorem 5.1 are listed in the following theorem.

Theorem 5.2. For the function $u$ from Theorem 5.1, it holds that

(i) $u \in L^{\infty}(I, V) \cap C^{0,1}\left(I, B_{2}^{1}(0,1)\right)$;

(ii) $u$ is strongly differentiable a.e. in $I$ and $d u / d t \in L^{\infty}\left(I, B_{2}^{1}(0,1)\right)$;

(iii) $u^{(n)}(t), \bar{u}^{(n)}(t) \rightarrow u(t)$ in $V$ for all $t \in I$;

(iv) $d u^{(n)} / d t \rightarrow d u / d t$ in $L^{2}\left(I, B_{2}^{1}(0,1)\right)$.

Proof. On the basis of estimates (4.37) and (4.38), uniform convergence statement from Theorem 5.1 and the continuous embedding $V \hookrightarrow B_{2}^{1}(0,1)$, the assertions of the present theorem are direct consequences of [3, Lemma 1.3.15].

Gathering all the obtained results, we are in position to state our existence theorem.

Theorem 5.3. There is a unique weak solution to the problem (1.8)-(1.11) in the sense of Definition 2.2, namely the limit function $u$ from Theorem 5.1.

Proof. In light of what precedes, the properties (i) and (ii) from Definition 2.2 are already fulfilled. Moreover, since $u^{(n)} \rightarrow u$ in $C\left(I, B_{2}^{1}(0,1)\right)$ when $n \rightarrow \infty$ and, by definition, $u^{(n)}(0)=U_{0}$, it follows that $u(0)=U_{0}$ holds in $B_{2}^{1}(0,1)$ so the initial condition (1.9) is also fulfilled. It remains to show that $u$ satisfies the integral equation (2.14). Integrating $(5.1)^{n}$ over $(0, t) \subset I$ and invoking the fact that $u^{(n)}(0)=U_{0}$, we get the approximation scheme:

$$
\left(u^{(n)}(t)-U_{0}, \phi\right)_{B_{2}^{1}}+\int_{0}^{t}\left(\bar{u}^{(n)}(\tau), \phi\right) d \tau=\int_{0}^{t}\left(\bar{f}^{(n)}\left(\tau, \bar{u}^{(n)}\left(\tau-\frac{T}{n}\right)\right), \phi\right)_{B_{2}^{1}} d \tau .
$$

To investigate the behavior of (5.22) as $n \rightarrow \infty$, we need some additional convergence statements. Since $u^{(n)}(t) \rightarrow u(t)$ in $V$ for all $t \in I$ and since for all fixed $\phi \in V$, the linear functional $v \mapsto(v, \phi)_{B_{2}^{1}}$ is bounded on $V$, we have

$$
\left(u^{(n)}(t), \phi\right)_{B_{2}^{1}} \longrightarrow(u(t), \phi)_{B_{2}^{1}}, \quad \forall t \in I
$$

On the other hand, in view of the assumed Lipschitz continuity of $f$, we have

$$
\begin{aligned}
& \left\|\bar{f}^{(n)}\left(\tau, \bar{u}^{(n)}\left(\tau-\frac{T}{n}\right)\right)-f(\tau, u(\tau))\right\|_{B_{2}^{1}} \\
& \quad=\left\|f\left(t_{j}, \bar{u}^{(n)}\left(\tau-\frac{T}{n}\right)\right)-f(\tau, u(\tau))\right\|_{B_{2}^{1}} \\
& \quad \leqslant l\left[\left|t_{j}-\tau\right|\left(1+\left\|u_{j-1}\right\|_{B_{2}^{1}}+\|u(\tau)\|_{B_{2}^{1}}\right)+\left\|\bar{u}^{(n)}\left(\tau-\frac{T}{n}\right)-u(\tau)\right\|_{B_{2}^{1}}\right],
\end{aligned}
$$


18 A semilinear heat equation with a nonlocal condition

for all $\tau \in\left(t_{j-1}, t_{j}\right], 1 \leqslant j \leqslant n$, whence

$$
\left\|\bar{f}^{(n)}\left(\tau, \bar{u}^{(n)}\left(\tau-\frac{T}{n}\right)\right)-f(\tau, u(\tau))\right\|_{B_{2}^{1}} \leqslant l\left(\frac{c_{13}}{n}+\left\|\bar{u}^{(n)}\left(\tau-\frac{T}{n}\right)-u(\tau)\right\|_{B_{2}^{1}}\right),
$$

for all $\tau \in(0, T]$, where $c_{13}:=T\left(1+c_{2}+\|u\|_{C\left(I, B_{2}^{1}(0,1)\right)}\right)$. But owing to the estimates (4.39), (4.40), and (5.2), we have

$$
\begin{aligned}
\left\|\bar{u}^{(n)}\left(\tau-\frac{T}{n}\right)-u(\tau)\right\|_{B_{2}^{1}} \leqslant & \left\|\bar{u}^{(n)}\left(\tau-\frac{T}{n}\right)-\bar{u}^{(n)}(\tau)\right\|_{B_{2}^{1}} \\
& +\left\|\bar{u}^{(n)}(\tau)-u^{(n)}(\tau)\right\|_{B_{2}^{1}}+\left\|u^{(n)}(\tau)-u(\tau)\right\|_{B_{2}^{1}} \\
\leqslant & \frac{2 c_{3} T}{n}+\frac{c_{12}}{n^{1 / 2}}, \quad \forall \tau \in(0, T]
\end{aligned}
$$

which in turn implies that

$$
\left\|\bar{f}^{(n)}\left(\tau, \bar{u}^{(n)}\left(\tau-\frac{T}{n}\right)\right)-f(\tau, u(\tau))\right\|_{B_{2}^{1}} \leqslant \frac{\left(c_{13}+2 c_{3} T+c_{12}\right) l}{n^{1 / 2}}, \quad \forall \tau \in(0, T]
$$

therefore

$$
\bar{f}^{(n)}\left(\tau, \bar{u}^{(n)}\left(\tau-\frac{T}{n}\right)\right) \underset{n \rightarrow \infty}{\longrightarrow} f(\tau, u(\tau)) \quad \text { in } B_{2}^{1}(0,1), \forall \tau \in(0, T] .
$$

Now, due to (5.7) and (5.9), the functions $\left|\left(\bar{f}^{(n)}\left(\tau, \bar{u}^{(n)}(\tau-T / n)\right), \phi\right)_{B_{2}^{1}}\right|$ and $\left|\left(\bar{u}^{(n)}(\tau), \phi\right)\right|$ are uniformly bounded with respect to both $\tau$ and $n$, so the Lebesgue theorem of dominated convergence may be applied to (5.28) as well as to the convergence statement (iii) from Theorem 5.2 giving

$$
\begin{gathered}
\int_{0}^{t}\left(\bar{f}^{(n)}\left(\tau, \bar{u}^{(n)}\left(\tau-\frac{T}{n}\right)\right), \phi\right)_{B_{2}^{1}} d \tau \longrightarrow \int_{0}^{t}(f(\tau, u(\tau)), \phi)_{B_{2}^{1}} d \tau, \quad \forall t \in I, \\
\int_{0}^{t}\left(\bar{u}^{(n)}(\tau), \phi\right) d \tau \longrightarrow \int_{0}^{t}(u(\tau), \phi) d \tau, \quad \forall t \in I,
\end{gathered}
$$

as $n \rightarrow \infty$. Then, passing to the limit $n \rightarrow \infty$ in (5.22), we obtain by (5.23), (5.29), and (5.30) that

$$
\left(u(t)-U_{0}, \phi\right)_{B_{2}^{1}}+\int_{0}^{t}(u(\tau), \phi) d \tau=\int_{0}^{t}(f(\tau, u(\tau)), \phi)_{B_{2}^{1}} d \tau,
$$

for all $\phi \in V$ and $t \in I$. Finally, differentiating this last identity with respect to $t$ recalling that $u: I \rightarrow B_{2}^{1}(0,1)$ is strongly differentiable for a.e. $t \in I$, we get the required relation 
(2.14) thanks to the relation

$$
\frac{d}{d t}(u(t), \phi)_{B_{2}^{1}}=\left(\frac{d u}{d t}(t), \phi\right)_{B_{2}^{1}}, \quad \forall t \in I, \forall \phi \in V .
$$

Thus $u$ weakly solves the problem (1.8)-(1.11).

Regarding the uniqueness, let us consider two weak solutions $\hat{u}$ and $\tilde{u}$ of (1.8)-(1.11). Subtracting the identity (2.14) written for $\tilde{u}$ from the same identity written for $\hat{u}$ and putting $\phi=\widehat{u}(t)-\tilde{u}(t)$ in the resulting relation, we get

$$
\left(\frac{d u}{d t}(t), u(t)\right)_{B_{2}^{1}}+\|u(t)\|^{2}=(f(t, \hat{u}(t))-f(t, \tilde{u}(t)), u(t))_{B_{2}^{1}}, \quad \forall t \in I
$$

where $u:=\hat{u}-\tilde{u}$. Then, integrating between 0 and $t$ by taking into account that $((d u /$ $d t)(t), u(t))_{B_{2}^{1}}=(1 / 2)(d / d t)\|u(t)\|_{B_{2}^{1}}^{2}$ and $u(0)=0$, we derive

$$
\begin{aligned}
\|u(t)\|_{B_{2}^{1}}^{2}+2 \int_{0}^{t}\|u(\tau)\|^{2} d \tau & =2 \int_{0}^{t}(f(\tau, \widehat{u}(\tau))-f(\tau, \tilde{u}(\tau)), u(\tau))_{B_{2}^{1}} d \tau \\
& \leqslant 2 \int_{0}^{t}\|f(\tau, \widehat{u}(\tau))-f(\tau, \tilde{u}(\tau))\|_{B_{2}^{1}}\|u(\tau)\|_{B_{2}^{1}} d \tau \\
& \leqslant 2 l \int_{0}^{t}\|u(\tau)\|_{B_{2}^{1}}^{2} d \tau, \quad \forall t \in I,
\end{aligned}
$$

from where Gronwall's lemma yields $\|u(t)\|_{B_{2}^{1}}^{2}=0$, for all $t \in I$, which means that $\hat{u}=$ $\tilde{u}$.

To conclude, we give a result of continuous dependence of the solution upon the data. Theorem 5.4. Let $f^{*}: I \times L^{2}(0,1) \rightarrow L^{2}(0,1)$ and $U_{0}^{*}:[0,1] \rightarrow \mathbb{R}$ be two given functions satisfying assumptions ( $\mathrm{H} 1)-(\mathrm{H} 3)$. If $u^{*}$ denotes the weak solution of problem (1.8)-(1.11) corresponding to the pair $\left(f^{*}, U_{0}^{*}\right)$ in lieu of $\left(f, U_{0}\right)$, then the inequality

$$
\begin{aligned}
& \left\|u(t)-u^{*}(t)\right\|_{B_{2}^{1}}^{2}+\int_{0}^{t}\left\|u(\tau)-u^{*}(\tau)\right\|^{2} d \tau \\
& \quad \leqslant\left\|U_{0}-U_{0}^{*}\right\|_{B_{2}^{1}}^{2}+\int_{0}^{t}\left\|f(\tau, u(\tau))-f^{*}\left(\tau, u^{*}(\tau)\right)\right\|_{B_{2}^{1}}^{2} d \tau
\end{aligned}
$$

holds for all $t \in I$.

Proof. Subtract identities (2.14) for $u$ and $u^{*}$, put $\phi=u(t)-u^{*}(t)$, and integrate the resulting relation over $(0, t)$. we have:

$$
\begin{gathered}
\frac{1}{2}\left\|u(t)-u^{*}(t)\right\|_{B_{2}^{1}}^{2}-\frac{1}{2}\left\|u(0)-u^{*}(0)\right\|_{B_{2}^{1}}^{2}+\int_{0}^{t}\left\|u(t)-u^{*}(t)\right\|^{2} d \tau \\
=\int_{0}^{t}\left(f(\tau, u(\tau))-f^{*}\left(\tau, u^{*}(\tau)\right), u(t)-u^{*}(t)\right)_{B_{2}^{1}} d \tau,
\end{gathered}
$$


hence

$$
\begin{aligned}
& \left\|u(t)-u^{*}(t)\right\|_{B_{2}^{1}}^{2}+2 \int_{0}^{t}\left\|u(t)-u^{*}(t)\right\|^{2} d \tau \\
& \quad \leqslant\left\|U_{0}-U_{0}^{*}\right\|_{B_{2}^{1}}^{2}+2 \int_{0}^{t}\left\|f(\tau, u(\tau))-f^{*}\left(\tau, u^{*}(\tau)\right)\right\|_{B_{2}^{1}}\left\|u(t)-u^{*}(t)\right\|_{B_{2}^{1}} d \tau .
\end{aligned}
$$

The application of (2.12) to the second term in the right-hand side leads to

$$
\begin{aligned}
& \left\|u(t)-u^{*}(t)\right\|_{B_{2}^{1}}^{2}+2 \int_{0}^{t}\left\|u(t)-u^{*}(t)\right\|^{2} d \tau \\
& \quad \leqslant\left\|U_{0}-U_{0}^{*}\right\|_{B_{2}^{1}}^{2}+\varepsilon \int_{0}^{t}\left\|f(\tau, u(\tau))-f^{*}\left(\tau, u^{*}(\tau)\right)\right\|_{B_{2}^{1}}^{2} d \tau+\frac{1}{\varepsilon} \int_{0}^{t}\left\|u(t)-u^{*}(t)\right\|_{B_{2}^{1}}^{2} d \tau,
\end{aligned}
$$

from which inequality (5.35) follows by taking $\varepsilon=1$. This achieves the proof.

\section{References}

[1] A. Bouziani, On the quasi static flexure of a thermoelastic rod, Communications in Applied Analysis 6 (2002), no. 4, 549-568.

[2] - On the solvability of parabolic and hyperbolic problems with a boundary integral condition, International Journal of Mathematics and Mathematical Sciences 31 (2002), no. 4, 201213.

[3] J. Kačur, Method of Rothe in Evolution Equations, Teubner-Texte zur Mathematik, vol. 80, BSB B. G. Teubner Verlagsgesellschaft, Leipzig, 1985.

[4] A. Kufner, O. John, and S. Fučík, Function Spaces, Noordhoff International, Leyden, 1977.

[5] N. Merazga and A. Bouziani, Rothe method for a mixed problem with an integral condition for the two-dimensional diffusion equation, Abstract and Applied Analysis 2003 (2003), no. 16, 899922.

Nabil Merazga: Département de Mathématiques, Centre Universitaire Larbi Ben M’hidi,

Oum El Bouagui 04000, Algeria

E-mail address: nabilmerazga@yahoo.fr

Abdelfatah Bouziani: Département de Mathématiques, Centre Universitaire Larbi Ben M’hidi, Oum El Bouagui 04000, Algeria

E-mail address: af_bouziani@hotmail.com 


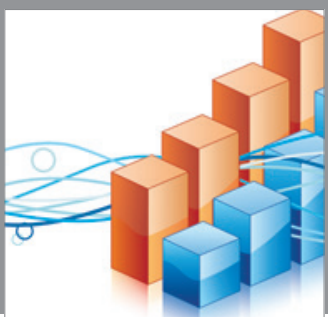

Advances in

Operations Research

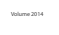

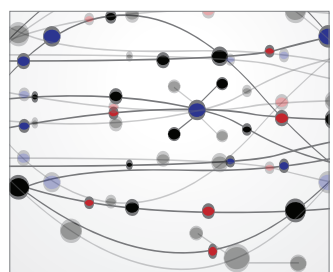

\section{The Scientific} World Journal
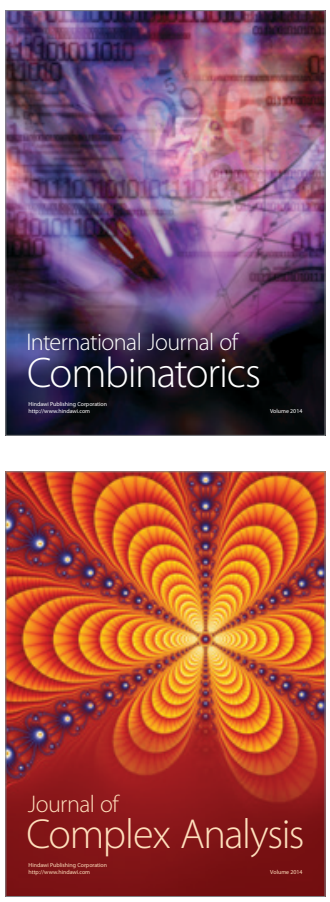

International Journal of

Mathematics and

Mathematical

Sciences
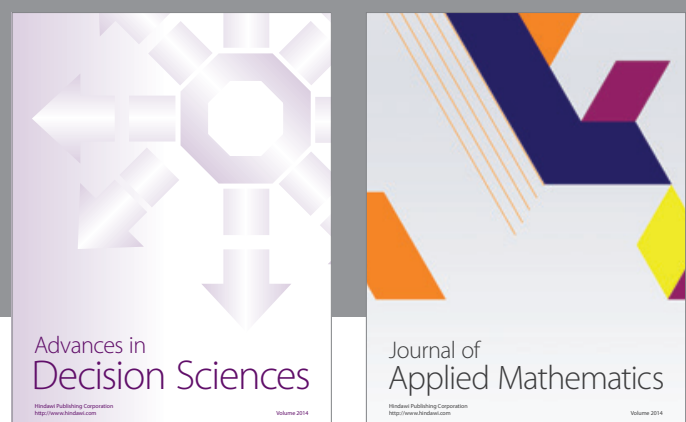

Journal of

Applied Mathematics
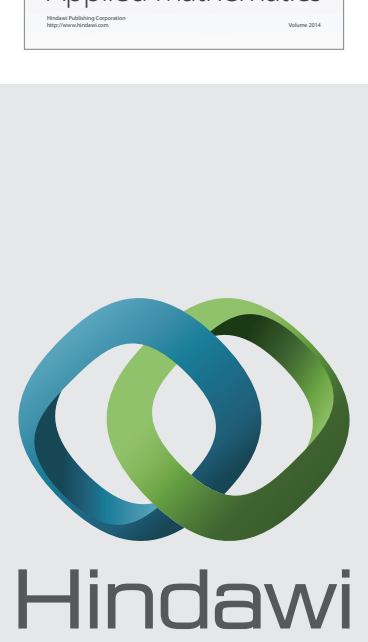

Submit your manuscripts at http://www.hindawi.com
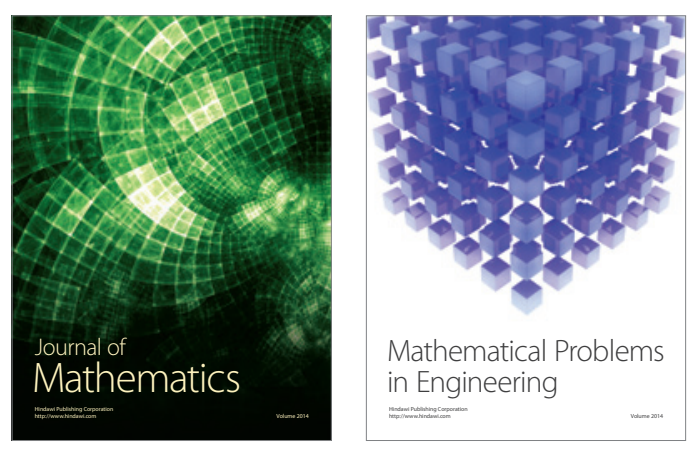

Mathematical Problems in Engineering
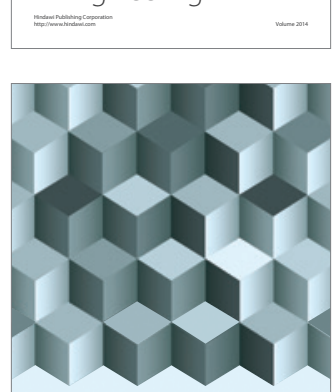

Journal of

Function Spaces
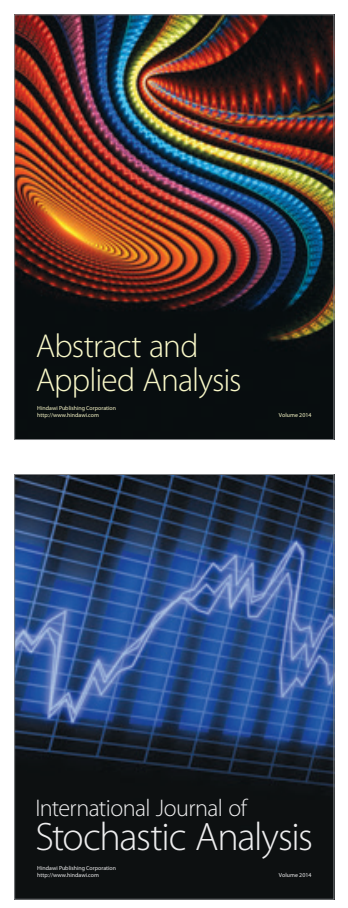

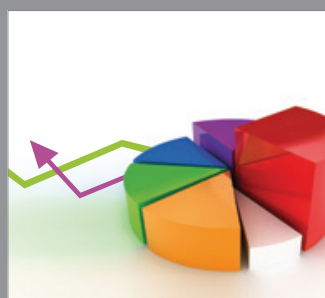

ournal of

Probability and Statistics

Promensencen
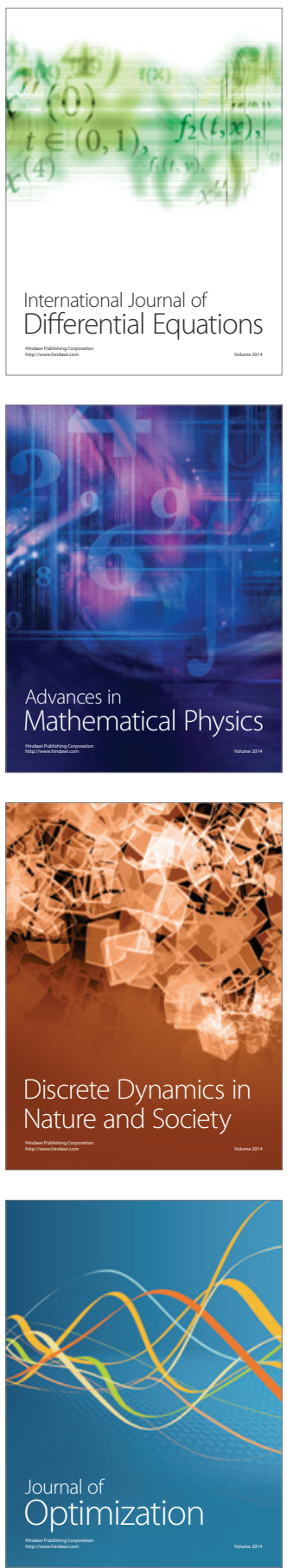